\title{
Tabularia
}

\section{La dispersion des bibliothèques du diocèse de Rouen au temps des invasions normandes : autour d'un manuscrit carolingien anciennement conservé à la Sainte-Trinité de Fécamp}

The Dispersion of Libraries in the Diocese of Rouen in the Days of Norman Raids: Apropos of a carolingian manuscript formely preserved at the Sainte-Trinité abbey in Fécamp

Jacques Le Maho

\section{OpenEdition}

Journals

Édition électronique

URL : http://journals.openedition.org/tabularia/968

DOI : 10.4000/tabularia.968

ISSN : 1630-7364

Éditeur :

CRAHAM - Centre Michel de Boüard, Presses universitaires de Caen

Référence électronique

Jacques Le Maho, « La dispersion des bibliothèques du diocèse de Rouen au temps des invasions normandes : autour d'un manuscrit carolingien anciennement conservé à la Sainte-Trinité de

Fécamp », Tabularia [En ligne], Écrire l'histoire au Moyen Âge, mis en ligne le 15 juin 2004, consulté le 02 mai 2019. URL : http://journals.openedition.org/tabularia/968 ; DOI : 10.4000/tabularia.968 


\title{
La dispersion des bibliothèques du diocèse de Rouen au temps des invasions normandes: autour d'un manuscrit carolingien anciennement conservé à la Sainte-Trinité de Fécamp
}

\section{The Dispersion of Libraries in the Diocese of Rouen in the Days of Norman Raids: Apropos of a carolingian manuscript formely preserved at the Sainte-Trinité abbey in Fécamp}

\author{
Jacques LE MAHO (CNRS) \\ CRAHM-UMR 6577 \\ Université de Caen Basse-Normandie
}

Résumé :

Propriété de l'abbaye de Fécamp jusqu'à la Révolution, le $m s 524$ de la bibliothèque de Rouen, écrit au IX siècle, renferme diverses œuvres relatives à l'astronomie et au calcul du temps. L'analyse de la notice obituaire d'Hardinus au fol. 78 semble confirmer l'hypothèse, déjà avancée par plusieurs auteurs, de sa réalisation par un moine de l'abbaye de Fontenelle/ Saint-Wandrille. On ignore comment l'ouvrage est arrivé à Fécamp. Parmi les différentes filières possibles, on examine ici celle du monastère de Blangy-sur-Ternoise au diocèse de Thérouanne, un des refuges des moines de Fontenelle au temps des invasions normandes, devenu prieuré de Fécamp en 1032.

Mots-clés: Blangy-sur-Ternoise, Fécamp, Fontenelle, manuscrit, Normands, Saint-Omer, Saint-Wandrille, scriptorium, Senlis, Thérouanne.

Abstract:

A property of the abbey of Fécamp until the French Revolution, the ms 524 of the Rouen Library, written in the 9th century, contains different works dealing with astronomy and computation. The analysis of the obituary notice of Hardinus on fol. 78 seems to confirm the hypothesis, already put forward by some authors, of its being writen by a monk of the abbey of Fontenelle/SaintWandrille. It is not known how the manuscript came to Fécamp. Among the different possible channels, this paper considers that of the Blangy-sur-Ternoise monastery in the diocese of Thérouanne, one the refuges of the Fontenelle monks during Norman raids, which became the priory of Fécamp in 1032.

Keywords: Blangy-sur-Ternoise, Fécamp, Fontenelle, manuscript, Normans, Saint-Omer, SaintWandrille, scriptorium, Senlis, Thérouanne.

Nul n'ignore que les manuscrits étaient jadis indispensables au fonctionnement des établissements religieux et qu'en conséquence, leur sauvetage était considéré 
comme une priorité majeure lors des exodes de la période des invasions normandes. On sait également qu'en quittant leurs refuges pour regagner leur monastère ou pour se mettre en quête d'un autre asile, il arrivait souvent aux réfugiés de manifester leur reconnaissance à leurs hôtes en leur laissant non seulement des reliques de leurs saints patrons, mais aussi un ou plusieurs ouvrages de leur scriptorium. Nombre de manuscrits passèrent ainsi d'une région à l'autre. Pour montrer que tout cela ne correspond nullement à un simple cliché historiographique, il nous suffira de rappeler le cas des moines de l'abbaye de Saint-Maixent, au diocèse de Poitiers, laissant des Vies de saint Maixent et de saint Léger dans leurs refuges de Bretagne, celui des moines d'Elnone ${ }^{1}$, abandonnant plusieurs manuscrits à Saint-Germain des Prés, ou encore le cas des religieux de l'abbaye de Landévennec, arrivés dans la place-forte de Montreuil-sur-Mer avec des malles pleines de livres. Cette mise en circulation d'une quantité considérable de manuscrits, venus quelquefois de contrées très lointaines, favorisa le cheminement d'influences culturelles dans les domaines les plus divers. Il a été démontré que la grande Bible de Saint-Martial de Limoges, réalisée au cours de la deuxième moitié du IX ${ }^{\mathrm{e}}$ siècle, présente des décorations d'inspiration tourangelle, ce qui s'explique aisément quand on sait que de nombreuses communautés monastiques de la région de la Loire moyenne vinrent trouver refuge en Auvergne et dans le Limousin ${ }^{2}$.

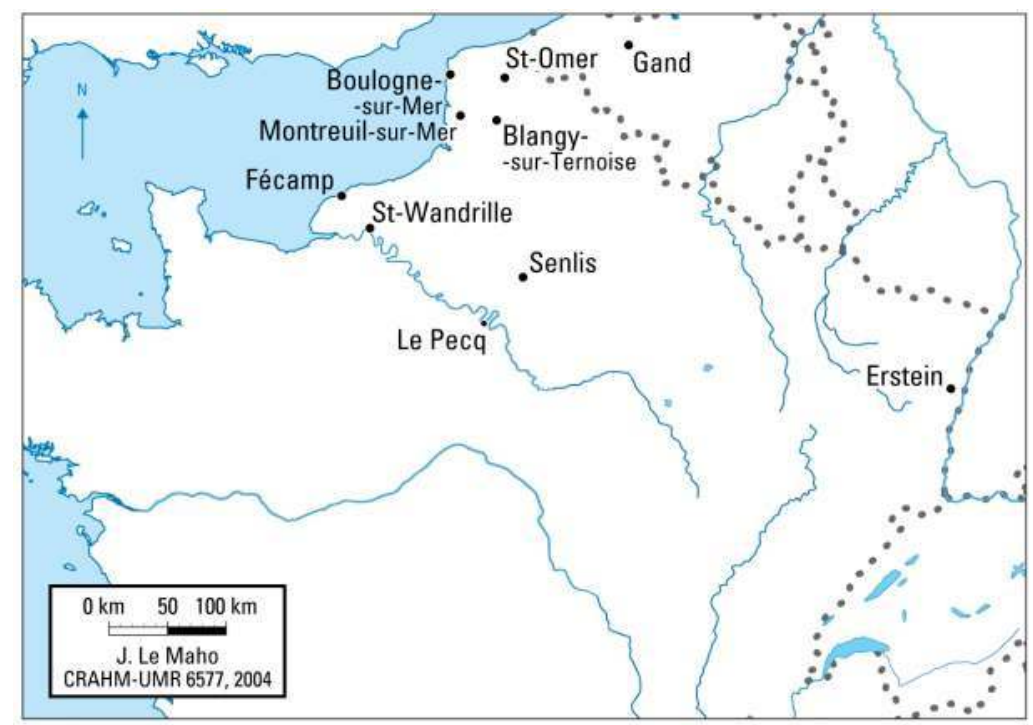

Fig. 1 : Carte des principaux lieux cités.

1. Dép. Nord, Saint-Amand-les-Eaux, chef-lieu de cant.

2. RICHÉ, Pierre, "Conséquences des invasions normandes sur la culture monastique dans l'Occident franc», in I Normanni e la loro espansione in Europa nell'Alto Medio Evo, Settimane di studio del Centro italiano di studi sull'alto medioevo, Spolète, 1969, p. 716 et suiv. 
Qu'en est-il pour la province ecclésiastique de Rouen, sachant qu'elle partage avec la Bretagne et les royaumes du nord-est de l'Angleterre la particularité d'avoir été, au terme de plusieurs décennies de raids et de pillages, pratiquement vidée de sa population monastique?

Il n'est sans doute pas nécessaire de revenir longuement sur la célèbre anecdote du «prêtre de Jumièges » qui, fuyant la Basse Seine où les Normands avait établi un camp en 857/858, traversa tout le pays et arriva au monastère de Saint-Gall, en Alémanie. Dans ses bagages, il avait un antiphonaire dont il fit cadeau à ses hôtes. L'ouvrage intéressa beaucoup les moines de Saint-Gall, car il contenait un type de notations musicales qui ne leur était pas familier et dont ils allaient se resservir par la suite $^{3}$. Moins connu, mais tout aussi intéressant pour l'histoire des échanges culturels, est le cas d'un lot de manuscrits ayant sans doute appartenu à l'église de Coutances et qui aboutit à Angers lors d'un exode provoqué par les mêmes événements de 857/858. Après le départ des clercs de Coutances, ces textes restèrent en possession des moines de Saint-Martin d'Angers. C'est ainsi que nous est parvenue une liste épiscopale de Rouen qui va des origines jusqu'au milieu du IX ${ }^{\mathrm{e}}$ siècle, et ce document est particulièrement précieux, car c'est le plus ancien catalogue de Rouen qui nous ait été conservé 4 . Parmi les textes normands échoués à Angers se trouvait également un recueil des actes d'un concile de la province de Rouen. Son existence nous est connue par la copie partielle qui fut réalisée au début du $\mathrm{X}^{\mathrm{e}}$ siècle par Réginon de Prüm pour une collection canonique ${ }^{5}$. On peut certes s'étonner qu'un auteur résidant au diocèse de Trèves ait pu avoir connaissance des actes d'un concile de cette lointaine région des bords de la Manche. Il s'est d'ailleurs trouvé plus d'un historien pour mettre en doute l'origine rouennaise des textes cités par Réginon. Mais c'est oublier qu'au IX ${ }^{\mathrm{e}}$ siècle, les moines de Saint-Sauveur de Prüm étaient précisément possessionnés à Angers, ce qui leur permettait de disposer d'informations de première main sur cette partie de la Francia ${ }^{6}$. Ceci accrédite l'origine rouennaise du document et lui confère du même coup une grande importance pour l'histoire

3. DuFT, Jean, «Le "presbyter de Gimedia » apporte son Antiphonaire à Saint-Gall », in Jumièges Congrès scientifique du XIII centenaire, t. 2, Fécamp, Durand, 1955, p. 925-936.

4. Angers, Bibl. mun., ms 266, utilisé par L. Duchesne, Fastes épiscopaux de l'ancienne Gaule, t. 2, $2^{\circ}$ édition, Paris, Fontemoing et Cie, 1910, p. 201 et suiv. ; le dernier nom de la liste est Wanilo, entré en charge entre 856 et 858 . Sur l'exode des clercs de Coutances à Angers, voir LE MAHO, Jacques, "Un exode de reliques dans les pays de la Basse Seine à la fin du IX siècle», Bulletin de la Commission Départementale des Antiquités de la Seine-Maritime, t. XLVI, 1998, p. 138-139.

5. RÉGINON DE PRÜM, Libellus de ecclesiasticis disciplinis et religione christiana, éd. Jacques-Paul MigNE, Patrologie Latine, XXXII, col. 298 et suiv.

6. URSEAU, Charles (Chanoine), Cartulaire noir de la cathédrale d'Angers, Paris, Picard, 1908, p. XIV et 37. Sur la méthode de Réginon et l'utilisation par celui-ci de sources angevines, voir Karl Ferdinand WERNER, "Zur Arbeitsweise des Regino von Prüm », in ID., Einheit der Geschichte - Studien zur Historiographie, Thorbecke, Sigmaringen, 1999, p. 136-156; nous remercions vivement Pierre Bauduin pour cette référence. 
ecclésiastique de notre province, car il ne nous a été conservé aucun autre recueil conciliaire pour la période prénormande.

De la même façon, on peut se demander si la célèbre chronique carolingienne des Gesta abbatum de Fontenelle et les principaux éléments de la production hagiographique prénormande de ce monastère seraient parvenus jusqu'à nous si l'abbaye n'avait pas été évacuée en 858. Les bâtiments avaient été brûlés par les Normands quatre ans plus tôt, mais, à la différence de Jumièges et de Saint-Ouen de Rouen, l'établissement avait pu, grâce au versement d'une rançon, échapper à la destruction lors du premier raid scandinave en mai $841^{7}$. On peut donc supposer qu'à la suite de cette première alerte, les moines avaient eu la possibilité de mettre leur trésor et leurs manuscrits en lieu sûr. Parfois, on procédait au déménagement de bibliothèques entières. À une date sans doute assez voisine de 875-876, les archevêques de Rouen firent transporter une grande quantité de livres dans ce qui allait devenir un de leurs principaux lieux d'exil, le castrum de Braine-sur-Vesle, au diocèse de Soissons ${ }^{8}$.

Une fois arrivés dans les refuges, certains manuscrits commençaient une nouvelle carrière, passaient de main en main, étaient recopiés, annotés ou réécrits. C'est ainsi que les moines de Saint-Rémi de Reims rédigèrent une nouvelle Vie de saint Marcoul, abbé fondateur de Nantus en Cotentin, dont les reliques se trouvaient depuis 906 dans leur cella de Corbény au diocèse de Laon ; le modèle leur fut fourni par la Vita primitive emportée par les moines de Nantus lors de l'attaque normande de 889/890 sur le Cotentin ${ }^{9}$. Au début des années 920, les clercs de la cathédrale de Bayeux repliés en Île-de-France réalisèrent une copie du martyrologe hiéronymien ${ }^{10}$. Ils l'enrichirent de notices propres aux saints de Bayeux, mais ils ajoutèrent aussi le nom d'un saint du pays de Loches où ils avaient précédemment trouvé refuge et les notices de plusieurs autres saints de la région parisienne. À ce stade de sa composition - il allait encore recevoir par la suite quelques additions dans le Sénonnais -, ce manuscrit nous livre ainsi un témoignage de premier ordre, non seulement sur les routes suivies par les clercs de Bayeux au cours de leurs différents exodes, mais encore sur leurs relations avec les églises locales et sur leurs contacts avec d'autres groupes en exil. Pour les clercs qui pouvaient y avoir accès ou en obtenir des copies,

7. Dom Jean LAPORTE (éd.), Les premières Annales de Fontenelle (Chronicon Fontanellense), Mélanges de la Société de l'Histoire de Normandie, Rouen-Paris, Société de l'Histoire de Normandie, 1951, p. 75.

8. C'est ce qui ressort d'une lettre adressée par l'auteur d'une Vita Romani à Hugues, archevêque de Rouen (942-989) : Acta Sanctorum, octobre, X, p. 92.

9. PolY, Jean-Pierre, "La gloire des rois et la parole cachée ou l'avenir d'une illusion ", in Religion et culture autour de l'an Mil - Royaume capétien et Lotharingie, Études réunies par Dominique IOGNAT-Prat et Jean-Charles PiCARD, Paris, Picard, 1990, p. 168-169; Le MAHO, "Un exode...», p. 147-149.

10. GrosJeAn, Paul, «Une source insulaire d'additions à un manuscrit du martyrologe hiéronymien ", Analecta Bollandiana, 65, 1947, p. 139-140. 
les manuscrits entreposés dans les refuges représentaient une incomparable source d'informations sur les usages liturgiques et les cultes des diocèses évacués. Mais, mal gardés, conservés dans des castella ou des monastères devenus les enjeux de luttes entre les dynasties princières, ces fonds étaient souvent l'objet de vols ou de pillages. Lorsque l'archevêque de Rouen Hugues (942-989) demanda à un certain Gerardus, abbé dans le Soissonnais, de réécrire la Vie de saint Romain de Rouen, Gérard se mit en quête de documentation et se rendit pour cela à Braine-sur-Vesle, espérant y trouver un exemplaire de la Vita s. Romani dans la bibliothèque de l'archevêque. Malheureusement, précise-t-il dans une lettre à son commanditaire, beaucoup d'ouvrages avaient été perdus «par négligence » et le livre recherché ne s'y trouvait plus ${ }^{11}$.

Nul ne saurait dire combien de manuscrits de la province de Rouen ont finalement survécu à la grande dispersion des $\mathrm{IX}^{\mathrm{e}}$ et $\mathrm{X}^{\mathrm{e}}$ siècles. Leur recensement, qui reste à réaliser, nécessiterait une enquête systématique à travers les bibliothèques d'une large partie de la France, de la Suisse et de l'Allemagne, et il va de soi qu'il ne faudrait pas s'attendre pour autant à des résultats spectaculaires. Compte tenu de l'obscurité presque complète qui règne sur la production textuelle de la future Normandie au haut Moyen Âge - le cas de Fontenelle constituant, à cet égard, une remarquable exception -, la moindre information pourrait cependant s'avérer précieuse. En dehors du problème de l'identification du scriptorium, la principale difficulté, celle à laquelle on se heurte par exemple pour un manuscrit recopié en 833 à l'abbaye de Deux-Jumeaux ${ }^{12}$, est d'établir son historique et de s'assurer qu'il s'agit bien d'un texte sorti au moment des invasions scandinaves. N'est-il pas plutôt le témoin d'un échange antérieur, comme ce fut probablement le cas du martyrologe de Fontenelle ${ }^{13}$, arrivé à Wissembourg ${ }^{14}$ au VIII siècle? Inversement, il est certain que nombre d'ouvrages furent composés ou transcrits dans les refuges, tel le bénédictionnaire dont l'archevêque de Rouen Francon (v. 912 - v. 919) fit la commande à l'évêque Adelhelm de Sées, résidant alors en son domaine épiscopal de Moussyle-Neuf, dans la région parisienne ${ }^{15}$.

Le dossier qui forme le sujet de la présente communication - celui du manuscrit 524 de Rouen, propriété de l'abbaye de Fécamp jusqu'à la Révolution - illustre assez

11. Vita s. Romani, Acta Sanctorum, octobre, X, p. 92.

12. Dép. Calvados, cant. Isigny-sur-mer, com. Deux-Jumeaux. Musset, Lucien, « Notes sur l'ancienne abbaye de Deux-Jumeaux», Bulletin de la Société des Antiquaires de Normandie, t. 53, 1955 et 1956, p. 408-409.

13. DuboIs, Dom Jacques, Les martyrologes du Moyen Âge latin, Typologie des sources du Moyen Âge occidental, fasc. 26, Turnhout, Brepols, 1978, p. 31.

14. Dép. Bas-Rhin, chef-lieu de cant.

15. Dép. Seine-et-Marne, cant. Dammartin-en-Goële. DELISLE, Léopold, « Notice sur un sacramentaire de l'église de Paris ", Mémoires de la Société Impériale des Antiquaires de France, t. 23, $3^{\text {e }}$ série, t. III, p. 165-171; comme Léopold Delisle l'a reconnu par la suite, l'Adelelmus captivus, auteur du bénédictionnaire, ne peut être que l'évêque de Sées (ID., "Mémoire sur d'anciens sacramentaires », Mémoires de l'Académie des Inscriptions et Belles-Lettres, t. XXXII, 1886, p. 211-218). 
bien les difficultés inhérentes à ce genre d'enquête, mais aussi l'extrême intérêt des informations que de tels textes sont susceptibles de nous fournir sur la production et la circulation des manuscrits. Le point de départ étant, en l'occurrence, le monastère de Fécamp, rappelons qu'une communauté féminine y fut installée au VIIe siècle. Elle se dispersa dès les premiers raids scandinaves, le site fut réoccupé à la fin du $\mathrm{IX}^{\mathrm{e}}$ siècle par un groupe de moines du diocèse de Coutances ${ }^{16}$, mais cette réoccupation fut éphémère, les lieux retournèrent à l'abandon et la vie monastique n'y fut rétablie qu'en 1001, par une communauté masculine, après une première restauration sous la forme d'une collégiale en 990 . Pour le problème qui nous occupe, l'essentiel se ramène donc à deux questions: de quel scriptorium ce manuscrit était-il donc issu et par quel cheminement finit-il par aboutir à Fécamp? Dans une riche étude publiée en 1963, dom Jean Laporte a proposé quelques éléments de réponse, sans toutefois, de son propre aveu, parvenir à une explication complètement satisfaisante en ce qui concerne l'origine du manuscrit et les circonstances de son entrée dans le fonds de Fécamp ${ }^{17}$. Sur ces deux derniers points, il nous est cependant apparu que les différentes pistes possibles n'avaient peut-être pas été toutes explorées.

\section{Le manuscrit 524 (I 49) de Rouen}

Cet ouvrage se présente comme un petit codex de 197 feuillets. À la suite du traité De ecclesiasticis officiis d'Isidore de Séville ( $†$ 636), on y trouve divers opuscules relatifs à l'astronomie, au comput et à la mesure du temps : le livre des Origines du même Isidore de Séville, qui comprend un traité sur les mathématiques et l'arithmétique, des questions d'astronomie (voir figure 2) et de comput pascal, un calendrier avec indications astronomiques et mention des jours égyptiens (fol. 76 à 81), des règles de comput pascal et des décisions de la hiérarchie à ce sujet, telles que la lettre de Proterius, patriarche d'Alexandrie, au pape Léon le Grand et celle du même Léon à l'épiscopat des Gaules et d'Espagne. Suivent des œuvres de Bède le Vénérable († 735) sur le comput ecclésiastique, son De Temporum Ratione et une partie de sa chronique. Le tout, dans une écriture que dom Laporte situe autour du milieu du IX siècle, paraît d'une seule main, sauf les additions au calendrier, une note en anglo-saxon sur la date de la fondation de Rome (fol. 95v) et peutêtre les Lettres de Proterius et de saint Léon.

Le manuscrit ne porte aucune indication explicite de provenance. En deux endroits, on y voit cependant apparaitre le nom d'un certain Hardinus, diacre puis prêtre, et comme plusieurs auteurs l'ont remarqué depuis longtemps, c'est probablement dans ces deux mentions que se trouve la clé du problème. La première mention se

16. LE MAHO, «Un exode... », p. 171-181.

17. Dom Jean LAPORTE, "Un manuscrit de Fécamp du IXe siècle écrit à Saint-Wandrille?», Revue Mabillon, $53^{\mathrm{e}}$ année, $3^{\mathrm{e}}$ série, $\mathrm{n}^{\circ} 211$, janvier-mars 1963, p. 31-38. 


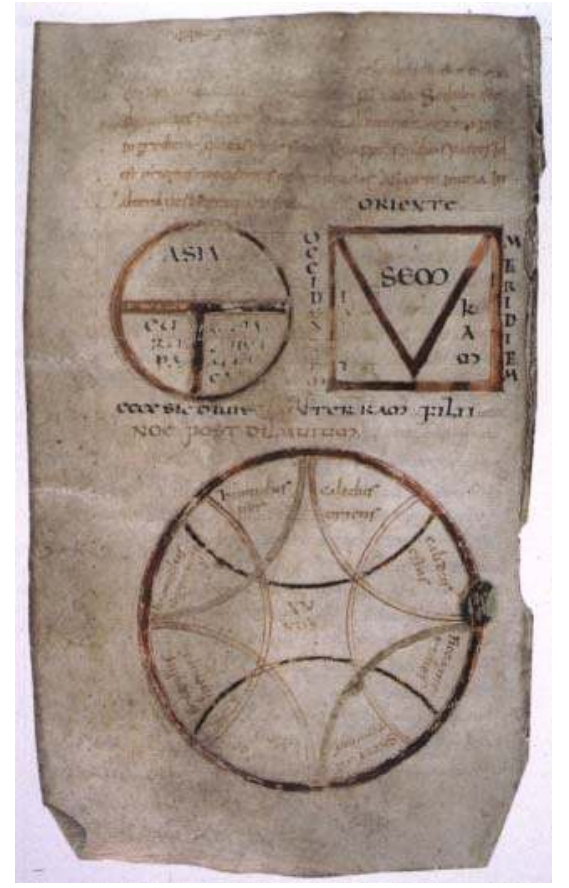

Fig. 2 : Rouen, ms 524 (I 49), fol. 74v, illustrations du traité d'astronomie (cliché Bibliothèque municipale de Rouen).

trouve dans le calendrier astronomique, à la date du 22 juin (fol. 76). Il s'agit d'une notice obituaire rédigée à la mémoire d'Hardinus, sacerdos. La seconde se présente sous la forme d'un colophon, c'est-à-dire d'une signature de copiste, à la fin du De temporibus de Bède le Vénérable: Explicit, deo juvante, Beda presbyter/Liber de Temporibus/Quiquis legerit, obsecro ut recordetur in suis orationibus/HARDINI INDIGNI DIACONI/qui scripsit summo cum labore librum hunc, ut Deus omnipotens cum bonis mansuris retribuet ei hujus laboris mercedem (fol. 171, voir figure 3 ).

Dans une lettre de 1891 à Léopold Delisle ${ }^{18}$, l'abbé Sauvage a été l'un des premiers à envisager une identification de cet Hardinus avec le célèbre Harduinus dont nous parlent les Gesta abbatum de l'abbaye de Fontenelle/Saint-Wandrille. Entré tout jeune dans ce monastère sous l'abbatiat d'Austrulf (747-753), Hardouin fut ordonné prêtre et il obtint l'autorisation de se retirer dans l'ermitage de Saint-Saturnin, sur le coteau nord du vallon de Fontenelle, pour y créer un scriptorium et y enseigner aux jeunes moines l'art de la calligraphie et l'arithmétique. Il mourut à Fontenelle en 811 , après avoir fait un pèlerinage à Rome et enrichi la bibliothèque

18. Lettre citée par Geneviève NORTIER, Les bibliothèques médiévales des abbayes bénédictines de Normandie, Caen, Caron, 1966, p. 7, note 4. 


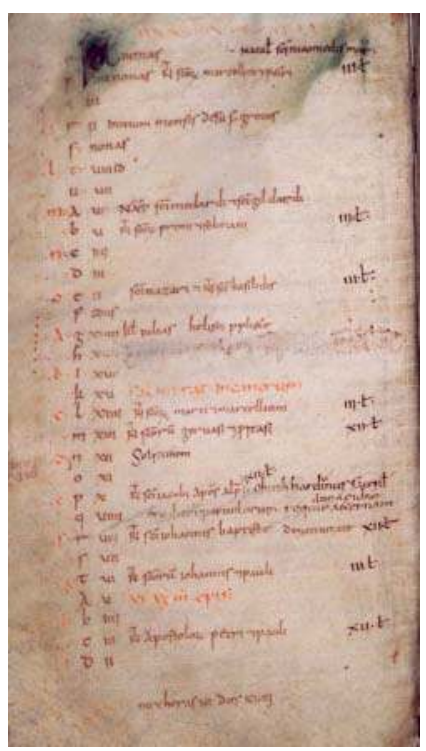

Fig. 3 : Rouen, ms 524 (I 49), fol. 78v, mention de l'obit d'Hardinus (cliché Bibliothèque municipale de Rouen).

de Fontenelle d'un grand nombre de livres transcrits de sa propre main ${ }^{19}$. Reprenant en 1963 le dossier du manuscrit de Rouen, dom Laporte a montré que plusieurs éléments viennent effectivement à l'appui d'une telle identification. C'est ainsi que trois opuscules figurant dans le codex fécampois, à savoir le traité d'arithmétique, les Lettres sur la question pascale et le De Temporibus de Bède, se retrouvent dans la liste des ouvrages que l'auteur des Gesta abbatum dit avoir été transcrits à Fontenelle par Hardouin en personne ${ }^{20}$. Dom Laporte fait également observer que dans le manuscrit de Fécamp, le texte de Bède présente certains caractères paléographiques dénotant une influence anglo-saxonne, ce qui pourrait indiquer l'utilisation d'un modèle en provenance directe d'Angleterre. Or, les moines de Fontenelle étaient bien placés pour obtenir des ouvrages anglo-saxons au temps d'Hardouin, car leur abbé Gervold (787-806), chargé par Charlemagne de l'administration des tonlieux

19. «Sub huius tempore bonae recordationis presbiter egregius nomine Harduinis florebat, qui in cella clari martyris Saturnini, quam beatus Vuandregisilus aedificaverat, ob gratiam vitae contemplativae remotior degens, quae sita in latere montis plagae aquilonalis praefatum coenobium spectat, plurimos arithmeticae artis disciplina alumnos imbuit ac arte scriptoria erudivit; erat enim in hac arte non mediocriter doctus. Unde plurima aecclesiae nostrae proprio sudore conscripta reliquit volumina... " (Gesta Sanctorum Patrum fontanellensis coenobii, éd. Fernand LOHIER et Jean LAPORTE, Rouen, Soc. de l'Hist. de Norm., 1936, p. 89-91; Chronique des abbés de Fontenelle (Saint-Wandrille), éd. Pascal Pradié, Paris, Les Belles Lettres, Les Classiques de l'Histoire de France, 283 p. (p. 140-143).

20. «... librum de aritmetica cum epistolis de ratione paschali,..., librum Bedae de naturis rerum ac temporibus volumen I...» (Gesta Sanctorum Patrum fontanellensis coenobii, éd. Fernand LOHIER et Jean LAPORTE, p. 90 ; éd. Pascal Pradié, p. 142-143). 
des ports de la Manche, était en relation d'amitié avec le roi Offa de Mercie, auprès de qui il conduisit à plusieurs reprises d'importantes missions diplomatiques ${ }^{21}$. Une autre possibilité est que le copiste lui-même ait été d'origine insulaire, ce qui dut être précisément le cas de nombreux moines de Fontenelle pendant toute la période prénormande ${ }^{22}$.

En dépit de ces multiples recoupements, dom Laporte conservait un léger doute sur l'identité de l'Hardinus du codex de Fécamp. «Il faut bien reconnaître », écritil, «que les éléments ainsi réunis peuvent permettre de parler de grande probabilité..., mais non de certitude. Nous n'avons pas de preuve claire et irréfutable de l'identité de cet Hardinus et de notre reclus de Saint-Saturnin ${ }^{23}$. On peut cependant penser que les dernières réticences de l'historien de Fontenelle eussent été levées si celui-ci avait eu la curiosité de lire l'intégralité de la notice nécrologique d'Hardinus au folio $78 \mathrm{v}$ (voir figure 4). Certains mots sont d'une encre très pâle, mais pas au point d'être indéchiffrables, et le texte se reconstitue comme suit: Obiith [sic] Hardinus sacerd (os)/eruditor parvulorum Dona ei D (omi) ne/requie ( $m$ ) aeternam. Geneviève Nortier avait fait déjà la même lecture et considéré à juste titre, nous semble-t-il, que ce prêtre qualifié d'eruditor parvulorum - professeur des jeunes moines - ne pouvait être autre qu'Hardouin de Fontenelle ${ }^{24}$.

Comme dom Laporte l'a bien montré, l'écriture du manuscrit est d'un type trop tardif pour que le colophon du fol. 171 - et par conséquent la transcription du De temporibus de Bède - puissent être de la main même d'Hardouin. Il s'agit donc de l'œuvre d'un copiste qui aurait tenu à reproduire la signature du maitre à la fin de l'ouvrage, tout en lui rendant hommage par la mise en exergue des trois mots HARDINI INDIGNI DIACONI, en grandes onciales rouges. La signature personnelle du copiste est vraisemblablement celle qui figure au-dessous, dans un texte acrostiche de huit lignes formant verticalement le nom d'homme Otbertus. Est-ce le même moine qui, en signe de vénération pour Hardouin, inscrivit son nom sur le calendrier? Deux détails pourraient appuyer cette hypothèse : l'orthographe Hardinus pour Harduinus, que l'on ne rencontre nulle part ailleurs dans les colophons

21. «Hic nempe Gervoldus super regni negotia procurator constituitur per multos annos, per diversos portus ac civitates exigens tributa ac vectigalia, maxime in Quentavvich. Unde Offae regi Anglorum sive Merciorum potentissimo, in amicitiis valde cognoscitur adjunctus. Extant adhuc epistolae ab eo ad illum, id est Gervoldum, directae, quibus se amicum ac familiarem illius carissimum fore pronuntiat. Nam multis vicibus ipse per se iussione invictisssimi regis Karoli ad praefatum regem Offam legationibus functus est» (Gesta Sanctorum Patrum fontanellensis coenobii, éd. Fernand LOHIER et Jean LAPORTE, p. 87 ; éd. Pascal Pradié, p. 136-139).

22. L'auteur des Gesta de Fontenelle a consacré toute une notice à l'un de ces religieux venus de la Britannia oceani insula, le moine-prêtre Baga, mort sous l'abbatiat de saint Bénigne (710-724): éd. Fernand Lohier et Jean LAPORTE, p. 27-28; éd. Pascal PradiÉ, p. 46-49.

23. LAPORTE, «Un manuscrit... », p. 35.

24. NORTIER, Geneviève, «Les bibliothèques médiévales... », p. 7. 


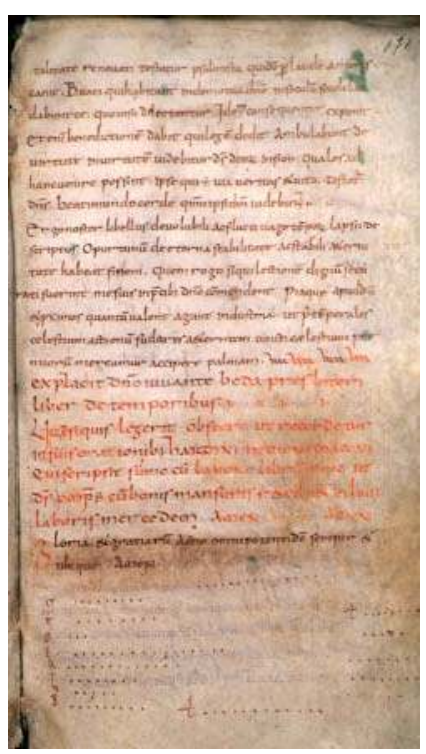

Fig. 4 : Rouen, ms 524 (I 49), fol. 171v, colophon d'Hardinus à la fin de l'ouvrage de Bède le Vénérable (cliché Bibliothèque municipale de Rouen).

du maître de Fontenelle ${ }^{25}$, et la forme obiith pour obiit, indicatrice d'une possible influence anglo-saxonne. Ajoutons que le calendrier contient également la mention obituaire de l'auteur du De temporibus, Bède le Vénérable; s'il n'est pas certain qu'elle soit de la même main, elle paraît cependant contemporaine de la notice d'Hardinus (fol. 78). Quoi qu'il en soit, il n'est pas douteux que le double hommage au maître de Fontenelle, assorti d'une précision biographique inédite sur celui-ci-le manuscrit de Fécamp est la seule source à indiquer le jour de sa mort, un 22 juin - ne peut venir que de moines de Saint-Wandrille. De même, si la mention de l'obit de Bède est relativement peu fréquente de ce côté-ci de la Manche, on notera qu'elle figure dans les Gesta abbatum fontanellensium ${ }^{26}$.

Au terme de cette première partie de l'analyse, il semble donc que nous disposions d'éléments suffisants pour considérer le manuscrit 524 de Rouen comme une production du scriptorium de l'abbaye de Fontenelle. Le copiste a dû travailler à une date assez largement postérieure à la mort d'Hardouin, mais la bibliothèque du monastère contenait encore à cette époque un certain nombre d'ouvrages transcrits de la main du maître et qui servaient de modèles aux moines du scriptorium. On

25. Sur trois colophons attribués à Harduin de Fontenelle dans des manuscrits carolingiens de Berlin et de Coblence, voir LAPORTE, «Un manuscrit... », p. 36-38; dans la notice obituaire du ms 524 de Rouen, quelqu'un a corrigé l'orthographe Hardinus en rajoutant un « $u$ » au dessus du nom.

26. Gesta Sanctorum Patrum fontanellensis coenobii, éd. Fernand LOHIER et Jean LAPORTE, p. 44 ; éd. Pascal PRADIÉ, p. 71. 
devait également y trouver un nécrologe de l'abbaye, avec mention de la date du décès d'Hardouin. Cependant, compte tenu de la marge d'imprécision inhérente à toute datation par la paléographie, en particulier pour les écritures postérieures au milieu du IX ${ }^{\mathrm{e}}$ siècle, nul ne saurait dire si le manuscrit fut réalisé à Fontenelle ou dans l'un des nombreux refuges ayant accueilli les moines après leur départ de la basse Seine en 858 . La seule indication chronologique que nous ayons trouvée parmi la première série d'additions au calendrier - une référence au culte des saints Médard et Gildard à Soissons (fol. 78v) - permet seulement d'assigner à ce remaniement une date postérieure à $841^{27}$.

Il n'en reste pas moins qu'il faut probablement orienter les recherches vers les exodes monastiques des $\mathrm{IX}^{\mathrm{e}}$ et $\mathrm{X}^{\mathrm{e}}$ siècles si l'on veut parvenir à savoir comment ce manuscrit de Saint-Wandrille a fini par aboutir un jour à Fécamp. C'est la démarche qui a été celle de dom Laporte, et elle l'a conduit à formuler deux hypothèses. La première se fonde sur l'histoire des reliques et des manuscrits de Fontenelle au début $\mathrm{du} \mathrm{X}^{\mathrm{e}}$ siècle. Comme on sait, la plus grande partie de cette collection était alors conservée à Boulogne-sur-Mer, et c'est de là qu'elle fut emmenée à Gand en 944 par Arnoul le Grand, comte de Flandre, sur le conseil de Gérard de Brogne. Il est d'autre part établi qu'aux environs de 950, le même Gérard de Brogne se rendit dans le diocèse de Senlis pour récupérer au nom d'Arnoul, en tant que dépositaire de l'héritage de Fontenelle, une ancienne propriété de l'abbaye, Rivecourt ${ }^{28}$. Selon une source normande du XI ${ }^{e}$ siècle, Gérard aurait poursuivi sa route jusqu'à Rouen où il aurait rencontré le duc Richard Ier pour lui demander restitution des domaines détenus par les descendants des compagnons de Rollon, mais ces derniers auraient refusé de s'en dessaisir ${ }^{29}$. Enfin, il est probable qu'un certain nombre de manuscrits de Fontenelle furent rapportés sur le site de l'abbaye-mère lorsqu'en 960, un groupe de moines de Gand, sous la conduite de Mainard, vint y rétablir la vie monastique; c'est ce qu'affirme au XI ${ }^{\mathrm{e}}$ siècle l'auteur de l'Inventio et Miracula sancti Vulfranni, et son témoignage est en l'occurrence fort plausible ${ }^{30}$. Dom Laporte s'est donc demandé si ces événements n'étaient pas susceptibles d'expliquer le retour en France de notre manuscrit: il aurait pu être rapporté, soit par Gérard de Brogne dans la région de l'Oise - ce qui justifierait la mention de Rieul, saint du diocèse de Senlis,

27. C'est le terminus post quem de la translation des reliques de saint Gildard de Rouen à Soissons. Sur cette question, voir LIFSHITZ, Felice, «The 'Exodus of holy bodies'reconsidered: the date of the translation of Gildardus of Rouen to Soissons ", Analecta Bollandiana, 110, 1992, p. 329-340.

28. Dép. Oise, cant. Estrées-Saint-Denis. Sur l'histoire de Fontenelle et le destin de ses reliques entre 858 et le milieu du $\mathrm{X}^{\mathrm{e}}$ siècle, la dernière mise au point est celle de VAN WERVEKE, Hans, "Saint-Wandrille et Saint-Pierre de Gand (IX ${ }^{\mathrm{e}}$ et $\mathrm{X}^{\mathrm{e}}$ siècles) », in Miscellanea Mediaevalia in memoriam Jan Frederik Niermeyer, Gröningen, J. B. Wolters, 1967, p. 79-92.

29. Inventio et miracula sancti Vulfranni, éd. Dom Jean LAPORTE, Mélanges de la Société de l'Histoire de Normandie, Rouen-Paris, 1938, p. 28-30.

30. «... libros quoque et cartas et quaedam ornamenta sed et filacteria cum preciosis sanctorum pigneribus $a b$ abbate suo et fratribus sibi indulta convexit...» (Inventio et miracula sancti Vulfranni, éd. Jean LAPORTE, p. 32-33). 
parmi les premières additions au calendrier (fol. 76v) -, soit directement par Mainard à l'abbaye de Fontenelle. Quel que soit le scénario retenu, cette hypothèse ne fournit cependant aucune explication à l'arrivée du manuscrit dans la bibliothèque de Fécamp. Dom Laporte a donc envisagé une seconde hypothèse où il fait entrer en ligne de compte les liens d'amitié ayant existé entre Fécamp et Fontenelle au début du IX $\mathrm{I}^{\mathrm{e}}$ siècle, liens très anciens puisqu'ils remontaient à l'époque de la fondation du monastère de femmes de Fécamp, faite sous la direction de l'abbé de Fontenelle, saint Wandrille en personne $(\dagger 668)^{31}$. Dans cette hypothèse, il faudrait supposer que les religieuses avaient reçu cet ouvrage en cadeau des moines de Fontenelle et qu'elles l'avaient emporté dans un de leurs refuges de la région picarde vers le milieu du IX $\mathrm{IX}^{\mathrm{e}}$ siècle. Dom Laporte imagine qu'il aurait pu ainsi aboutir à Nogent-lesVierges $^{32}$, non loin de Senlis - d'où la mention de saint Rieul -, et ensuite passer de là à l'abbaye de Fécamp, cette dernière ayant reçu en donation une partie du même domaine de Nogent au début du $\mathrm{XI}^{\mathrm{e}}$ siècle ${ }^{33}$. Compte tenu de l'obscurité totale qui entoure le sort de la communauté de Fécamp et des reliques de sa première abbesse Hildemarque après le milieu du $\mathrm{IX}^{\mathrm{e}}$ siècle ${ }^{34}$, ce scénario nous paraît cependant bien fragile. On pourra surtout objecter que le nom de Nogent-les-Vierges se rapporte à la présence des reliques de deux saintes de Touraine, sainte Maure et sainte Brigide, et qu'en dépit des apparences, il n'a donc aucun lien avec l'histoire du monastère féminin de Fécamp ${ }^{35}$.

\section{La piste de Blangy-sur-Ternoise}

Dans un texte du $\mathrm{X}^{\mathrm{e}}$ ou du $\mathrm{XI}^{\mathrm{e}}$ siècle contenant la relation des miracles de sainte Berthe, fondatrice au début du VIII ${ }^{\mathrm{e}}$ siècle du monastère de femmes de Blangy-surTernoise ${ }^{36}$, près d'Hesdin, il est fait mention du séjour que «certains moines ", fuyant les Vikings, auraient effectué dans ce monastère à l'invitation de l'abbesse Hersende ; arrivés au temps d'un «empereur Charles » avec les corps de leurs deux saints patrons, «Ansbert et Wandrille», ils seraient restés une vingtaine d'années à Blangy ${ }^{37}$.

La mention des reliques des saints Wandrille et Ansbert ne permet pas de douter que les quidam monachi de ce récit étaient des moines de Fontenelle: c'est avec les

31. Vita IIa Wandregisili, Acta Sanctorum, juillet, V, p. 276-277.

32. Dép. Oise, com. de Nogent-sur-Oise.

33. Dom Jean LAPORTE, «Un manuscrit... », p. 36.

34. On sait seulement que les restes du fondateur du monastère, le comte Waninge, reposaient dans la région de Ham (arr. Péronne, Somme) au Xe siècle. Voir sur ce point FOURNÉE, Jean, « Le culte populaire des saints fondateurs d'abbayes prénormandes ", in Les abbayes de Normandie - Actes du XIII Congrès des sociétés historiques et archéologiques de Normandie, 1979, p. 78.

35. Sur l'une des légendes associées à ces saintes, voir la Chronique de Robert de Torigni, éd. Léopold Delisle, Rouen, Société de l'Histoire de Normandie, t. 2, p. 147-148. Le lieu d'origine de la dévotion est Sainte-Maure-de-Touraine (dép. Indre-et-Loire, chef-lieu de cant.).

36. Dép. Pas-de-Calais, cant. Le Parcq. 
mêmes corps saints, tous deux exhumés du chœur de leur abbatiale en 858, que ces moines fuyant les Normands voyagèrent de refuge en refuge jusqu'au début du $\mathrm{X}^{\mathrm{e}}$ siècle $^{38}$. Cela étant, la narration comporte des difficultés chronologiques qui ont suscité les réserves de plus d'un auteur. En effet, l'épisode se place logiquement après juin 868 , puisque les moines de Fontenelle se trouvaient encore à cette date à Montreuil-sur-Mer, et avant le début des années 890 si c'est bien à ce moment que les moniales de Blangy quittèrent leur abbaye pour se réfugier en Alsace, à Erstein ${ }^{39}$. Cela correspondrait assez bien à une arrivée des moines sous le règne de Charles le Chauve (841-877) et à un séjour d'une vingtaine d'années à Blangy. Cependant, nous savons qu'au début des années 880 , l'invasion par les Normands de la zone située entre la Somme et l'Escaut provoqua une évacuation générale vers le sud. Il semble que la communauté de Fontenelle se soit alors repliée sur ses vastes propriétés de l'Ouest parisien, Le Pecq et ses dépendances ${ }^{40}$. À l'annonce de la grande flotte normande remontant la Seine vers Paris en novembre 885, les moines s'enfuirent à Chartres où ils trouvèrent refuge dans un monastère suburbain, puis dans un emplacement mis à leur disposition par l'évêque local à l'intérieur de la cité. Dans ces conditions, on peut effectivement concevoir quelques doutes, sinon sur la réalité du passage des moines de Fontenelle à Blangy, du moins sur le fait qu'ils soient restés vingt ans dans ce monastère.

Le problème se complique si l'on y ajoute une série de témoignages relatifs au passage des moines de Fontenelle dans un autre monastère du Ternois, celui de SaintOmer ${ }^{41}$, à environ $35 \mathrm{~km}$ de Blangy. Il s'agit tout d'abord d'un inventaire des reliques de cette abbaye, établi vers 1120 par Lambert, chanoine de Saint-Omer, d'après un document du XI ${ }^{\mathrm{e}}$ siècle; il mentionne entre autres les noms des saints Bavon, Wandrille, Ansbert, Wulfran et Winnoc, de sainte Austreberthe; la dernière mention pourrait se rapporter une translation de reliques depuis Montreuil-sur-Mer ou Marconne ${ }^{42}$ où s'étaient réfugiées les religieuses de Pavilly au diocèse de Rouen ${ }^{43}$. À cela s'ajoute le remarquable manuscrit 764 de la bibliothèque de Saint-Omer, contenant une partie

37. "Eodem quoque tempore, Normannica gente Galliam vastante, multisque Sanctorum locis in desolationem cadentibus, contigit quosdam monachos, propter eosdem paganos de suo fugere monasterio: ac sanctum Ansbertum et sanctum Wandregisilum transportare: venientes igitur ad monasterium sanctae Bertae, ubi veneranda Hersendis praeesse abbatissa videbatur, cum omni charitatis humanitate ab ea suscepti sunt : ibique viginti annis hospitabantur» (Miracula et Translatio Sanctae Berthae, A.A.S.S., Juillet II, 1721, p. 55). Le texte est suivi de la relation d'un miracle attribué aux saints Ansbert et Wandrille.

38. Thiron, dom Joseph, "Les reliques de saint Wandrille», L'abbaye Saint-Wandrille de Fontenelle, Noël 1970, p. 4-12

39. Dép. Bas-Rhin, chef-lieu de cant.

40. Dép. Yvelines, chef-lieu de cant. Sur ces propriétés de l'abbaye de Fontenelle, voir Lot Ferdinand, Études critiques sur l'abbaye de Saint-Wandrille, Paris, Honoré Champion, 1913, p. XXIII.

41. Dép. Pas-de-Calais, chef-lieu de cant.

42. Montreuil-sur-Mer ou Marconne: Dép. Pas-de-Calais, cant. Hesdin.

43. Chronicon de Gestis Normannorum in Francia, éd. Georg Heinrich PerTZ, in M.G.H. Scriptores, Hanovre, Hahn, 1826, t. 1, p. 532 et suiv. 
de l'office de saint Wandrille (fol. 1-3), sa généalogie (fol. 3v), puis sa biographie accompagnée d'enluminures et les miracles du même saint, et enfin une Vie en prose et en vers de saint Ansbert. Ces opuscules sont de plusieurs mains et leur réalisation se situe vraisemblablement en deux temps, début et second quart du $\mathrm{X}^{\mathrm{e}}$ siècle, mais celle-ci n'a pu évidemment se faire qu'à partir de manuscrits de Fontenelle alors présents dans la bibliothèque de Saint-Omer. Le doute n'est plus permis quand on rapproche ces données de la découverte de Claude Carozzi sur l'origine du texte légendaire de la Vita Dagoberti, censé retracer la biographie du roi Dagobert III $(† 715)$. Son étude publiée en 1984 semble en effet avoir établi de manière définitive que le texte en question fut écrit à Saint-Bertin/Saint-Omer par un moine de Fontenelle qui en reçut la commande vers 898-899 et se servit pour cela de plusieurs ouvrages issus du scriptorium de Saint-Wandrille, dont les Gesta abbatum, la Vita Wandregisili IIa et la généalogie de saint Wandrille, ces deux derniers correspondant précisément à deux sources du manuscrit 764 de Saint-Omer ${ }^{44}$. En complément à l'étude de Claude Carozzi, signalons enfin qu'une ancienne chronique de Fontenelle aujourd'hui perdue, mais dont il nous reste une brève analyse du XVII ${ }^{e}$ siècle, évoque le transfert des reliques de saint Wandrille et de saint Ansbert dans le monastère de Saint-Omer vers 899, à l'initiative d'un abbé Eucher et du «préfet» Erkanger, bien connu par ailleurs comme comte de Boulogne ${ }^{45}$. Dom Laporte, qui avait été le premier à attirer l'attention sur cette source en 1960, la tenait pour assez suspecte, mais les nouveaux éléments apportés depuis lors par Claude Carozzi lui confèrent en définitive une certaine crédibilité, du moins sur certains points comme le séjour des moines de Fontenelle à Saint-Omer vers la fin des années 890 .

Or, il semble que des liens étroits aient existé entre Blangy et Saint-Omer. L'une des églises du groupe monastique de Blangy était en effet placée sous le vocable de Saint-Omer, et selon l'auteur de la Vie de sainte Berthe, elle était celle des moines assurant les services religieux pour les moniales ${ }^{46}$. Deux hypothèses en découlent : ou l'ensemble de la communauté de Fontenelle avait trouvé refuge dès le départ à Saint-Omer, se contentant d'envoyer quelques moines à Blangy pour la desserte de ce monastère, ou alors l'épisode se décompose en deux temps: un séjour à Blangy entre 876 et le début des années 880, un repli sur Saint-Omer au retour de Chartres. Cette dernière hypothèse s'accorderait mieux avec la tradition d'une présence des reliques des abbés de Fontenelle à Blangy et avec la date alléguée de l'arrivée des moines à Saint-Omer. Elle expliquerait mieux aussi le choix de Saint-Omer. Muni

44. CarrozI, Claude, «La vie de saint Dagobert de Stenay: histoire et hagiographie », Revue Belge de Philologie et d'Histoire, t. LXII, 1984-2, p. 225-258.

45. Dom Jean LAPORTE, "Saint Gérard de Brogne à Saint-Wandrille et à Saint-Riquier », Revue Bénédictine, LXX, 1960, p. 143-146.

46. "Construxit nempe unam, extra portam, ecclesiam in honore sancti Audomari confessoris, ubi sacerdotes vel clerici ipsius coenobii divinum persolverent officium» (Vie de sainte Berthe, Acta Sanctorum, jul. t. II, 1867, p. 47-60). 
de solides défenses en 890 , ce monastère était susceptible d'offrir une certaine sécurité au groupe de réfugiés ${ }^{47}$, ce qui ne devait pas être le cas de Blangy où rien n'indique le maintien d'une présence monastique après 891 . Il n'est d'ailleurs nullement certain que les religieuses de Blangy se soient réinstallées dans leur abbaye au retour du grand exode du début des années 880 . On peut supposer que la communauté avait trouvé refuge dans le Bassin parisien comme beaucoup d'autres - serait-ce à l'origine de la présence d'une relique de sainte Berthe à Saint-Frambourg de Senlis ${ }^{48}$ et, par suite, de la mention de saint Rieul au fol. 76 de notre manuscrit ? - et qu'en raison de la persistance de la menace normande dans le Ternois en 891, elle prit directement le chemin de la vallée du Rhin.

Si nous nous sommes attardés sur cet épisode de l'exil des moines de Fontenellesans parvenir, toutefois, à percer toutes ses zones d'ombres -, c'est qu'il fournit peutêtre un élément de réponse à l'une des questions posées au début de notre enquête : comment le manuscrit 524 est-il parvenu jusqu'à Fécamp ? Il se trouve en effet qu'au début des années 1030, l'abbaye de Blangy-sur-Ternoise fut relevée par Roger, comte de Saint-Pol ${ }^{49}$ et que ce dernier en fit don aux moines bénédictins de la Sainte-Trinité de Fécamp pour qu'ils y établissent un prieuré ${ }^{500}$. À partir de ce moment, il est probable qu'un certain nombre de manuscrits de Blangy ont été transférés en Normandie pour y être copiés ou pour venir accroître le fonds, en cours de constitution, de la grande bibliothèque monastique de Fécamp. Nous savons de façon certaine que ce fut le cas pour au moins l'un d'entre eux, la Vie de sainte Berthe, dont les moines de Fécamp ont possédé deux exemplaires au XI ${ }^{\mathrm{e}}$ siècle; elle figurait parmi les ouvrages lus au réfectoire ${ }^{51}$. Dans ces conditions, il n'est pas interdit de supposer que notre manuscrit ait été envoyé, lui aussi, du Ternois. Ceci rejoindrait l'analyse de Geneviève Nortier, qui jugeait peu probable que l'ouvrage ait pu passer à Fécamp avant «l'installation des bénédictins au XI ${ }^{e}$ siècle » ${ }^{52}$.

C'est en effet au cours de cette période que le calendrier des folios 76 à 81 reçut la plupart de ses additions. On y retrouve tout le cortège des saints fécampois, ceux du cycle de Dijon comme sainte Paschasie (fol. 76) et saint Bénigne (fol. 81), mais aussi sainte Scholastique (fol. 76) et saint Benoît (fol. 79) - tous deux évidemment

47. FOURNIER, Gabriel, Le château dans la France médiévale - Essai de sociologie monumentale, Paris, Aubier Montaigne, 1978, p. 271-273.

48. Sacramentaire de Senlis, vers 880 (Paris, Bibliothèque Sainte-Geneviève, ms 111).

49. Dép. Pas-de-Calais, com. Saint-Pol-sur-Ternoise.

50. Gallia Christiana, t. XI, col. 206.

51. L'un des deux exemplaires fécampois de la Vita s. Berthae, celui de la Bibliothèque Vaticane, Reg. Lat. 755 (ff. 100-105), a été étudié par François AVRIL « Notes sur quelques manuscrits bénédictins normands du XI ${ }^{\mathrm{e}}$ et du XII ${ }^{\mathrm{e}}$ siècle ", Mélanges d'archéologie et d'Histoire, École française de Rome, t. LXXVI (1964), 2, p. 523-525. Le second exemplaire est conservé à la bibliothèque de Rouen (U3, ms 1400, fol. 90v-91v). Voir également GRÉMONT, Denis-Bernard, «Lectiones ad prandium à l'abbaye de Fécamp au XIII siècle », Cahiers Léopold Delisle, 20, 1971, p. 10.

52. NORTIER, «Les bibliothèques médiévales... », p. 7. 
postérieurs à l'arrivée de Guillaume de Volpiano en 1001 -, saint Taurin, patron de l'abbaye d'Évreux rattachée à Fécamp en 1034/1035 (fol. 79v), saint Cuthman (fol. 77v) qui était honoré à Steyning (Sussex, Angleterre), domaine entré dans le patrimoine de Fécamp entre 1066 et 1085, ou encore saint Apollinaire (fol. 79), dont le culte fut probablement introduit à Fécamp par Jean de Ravenne, abbé de 1028 à $1079^{53}$. On notera que la seule mention de saint Wandrille (fol. 79) est de la même main que ces additions du XIe siècle et que l'on ne trouve ni le nom de saint Ansbert, ni celui de sainte Berthe. Il n'y a cependant pas lieu de s'étonner de ces lacunes si l'on considère que le calendrier avait été conçu au départ pour servir au calcul astronomique - avec numérotation des jours, table de concordance, indication des cycles lunaires, etc. - et qu'il fut principalement utilisé comme tel au IX ${ }^{\mathrm{e}}$ siècle.

Faut-il en conclure que le manuscrit 524 provient en droite ligne de la bibliothèque carolingienne de Blangy? Étant donné que le site du monastère primitif demeura longtemps à l'abandon - le silence à ce sujet de l'acte de restauration de 1032 ne saurait constituer la preuve du contraire ${ }^{54}$-, une telle éventualité n'est possible qu'à condition d'admettre un retour d'Alsace de certains manuscrits. L'hypothèse est parfaitement concevable si l'on accorde foi au récit du $\mathrm{XI}^{\mathrm{e}}$ siècle évoquant le rapatriement du corps de sainte Berthe à Blangy ${ }^{55}$. Le narrateur s'attarde peu sur le sujet, mais on croit comprendre que cet épisode est sensiblement antérieur à la fondation du prieuré bénédictin, ce qui voudrait dire que le site avait été l'objet d'une première restauration avant 1032, peut-être sous la forme, très habituelle en pareil cas, d'une collégiale $^{56}$. Il paraît certain, d'autre part, que l'auteur des Miracula de sainte Berthe eut accès à des documents d'archives d'Erstein, et donc que des contacts ou des échanges ont existé entre le monastère alsacien et Blangy. Une autre hypothèse serait que

53. Sur le rattachement à Fécamp de Saint-Taurin d'Évreux, voir FAUROUX, Marie, Recueil des actes des ducs de Normandie, Mémoires de la Société des Antiquaires de Normandie, t. XXXVI, Caen, 1961, $\mathrm{n}^{\circ}$ 87; sur la donation de Steyning, voir BATES, David, Regesta Regum Anglo- Normannorum - The acta of William I (1066-1087), Oxford, Clarendon Press, 1998, $\mathrm{n}^{\circ} 141$; tous ces cultes se retrouvent dans le sanctoral de Fécamp récemment édité par David CHADD, The Ordinal of the abbey of the Holy Trinity Fécamp, Parts II, III \$ IV, Londres, Henry Bradshaw Society, 2002, passim.

54. Cet argument a silentio est un de ceux qu'avance Cassandra Potts pour soutenir la thèse d'une survivance de la vie monastique à Sainte-Berthe de Blangy à travers toute la période des invasions normandes (POTTS, Cassandra, «When the Saints Go Marching: Religious Connections and the Political Culture of Early Normandy", in Anglo-Norman Political Culture and the 12 th-Century Renaissance, Warren C. Hollister (éd.), Woodbridge, Boydell Press, 1997, p. 28). Dans le sillage des travaux de Felice LIFSHITZ, l'école américaine a défendu avec vigueur, depuis une quinzaine d'années, l'idée d'un faible impact de ces événements sur le destin des monastères de la Francia. Nous ne pouvons souscrire à cette théorie que contredisent à chaque instant l'évidence des faits aussi bien que les témoignages des contemporains. Pour Blangy, on observera que Roger de Saint-Pol est présenté dans une charte de Fécamp comme l'homme qui a relevé de ses ruines un lieu «diu destructum atque desolatum» (Musée de la Bénédictine, charte $\mathrm{n}^{\circ}$ 5).

55. Miracula s. Berthae, Acta Sanctorum, Jul. II, 4 juillet, p. 55-57.

56. D'après la même source, les reliques de sainte Berthe auraient été rapportées d'Alsace par deux clerici, Albuin et Ebroin (ibid.). 
notre manuscrit soit, en fait, resté depuis la fin du $\mathrm{IX}^{\mathrm{e}}$ siècle dans une bibliothèque $\mathrm{du}$ Ternois - on pense naturellement en premier lieu à celle de Saint-Omer, où se trouvaient plusieurs ouvrages de Saint-Wandrille - et qu'il soit parvenu à Blangy par cette voie plus directe: rappelons qu'il n'y a pas plus de $35 \mathrm{~km}$ entre Saint-Omer et Blangy. Quoi qu'il en soit, il est clair que le manuscrit provient plutôt du nord de la Francia que de l'autre source de textes à laquelle on est en droit de songer pour Fécamp après 1001, la Bourgogne. Avant son passage à la bibliothèque de Fécamp, il fut en effet l'objet d'une note additive en langue anglo-saxonne sur la date de la fondation de Rome (fol. 95v), peut-être écrite par un des nombreux clercs anglais qui séjournaient dans le Boulonnais et le Ternois au $\mathrm{X}^{\mathrm{e}}$ et au début du $\mathrm{XI}^{\mathrm{e}}$ siècle.

\section{Une enquête à poursuivre}

Si l'on admet que le manuscrit 524 de Rouen entra dans le fonds de Fécamp à la suite de la donation du prieuré de Blangy, on peut logiquement penser qu'il ne fut pas le seul à arriver par cette filière. En évoquant le cas du manuscrit 524, Geneviève Nortier se demandait s'il ne fallait pas attribuer la même origine à un évangéliaire de Fécamp conservé à la Bibliothèque nationale de France sous la double cote lat. 281 et 298. Écrit au VIII siècle, dans une belle écriture onciale, cet ouvrage est vraisemblablement «issu d'un scriptorium où s'exerçait l'influence anglo-saxonne», ce qui, comme on l'a vu plus haut, était le cas de l'atelier de Fontenelle. Geneviève Nortier n'a pas manqué non plus de faire le rapprochement avec le passage des Gesta abbatum de Fontenelle mentionnant parmi les ouvrages copiés par le maître Hardouin - celuilà même dont le colophon et l'obit figurent sur le manuscrit 524 - un volume des quatre Évangiles en littera romana ${ }^{57}$. Serait-ce précisément l'ouvrage de Fécamp? Le type de l'écriture correspond bien à l'époque d'Hardouin, c'est-à-dire à la seconde moitié du VIII' siècle, mais en l'absence de colophon (le manuscrit comporte d'importantes lacunes), il est impossible de se prononcer. En l'état actuel des recherches, on devra donc se contenter de porter ce volume sur la liste de ceux qui sont susceptibles - tout au plus - d'avoir transité par Blangy ou Saint-Omer avant d'atterrir à Fécamp.

C’est également vers un établissement religieux du nord de la France que l'on serait tenté de rechercher l'origine d'un autre manuscrit de Fécamp, le Codex Bigotianus, aujourd'hui conservé à la Bibliothèque nationale de France sous la cote lat. 3182. Écrit au début du $\mathrm{X}^{\mathrm{e}}$ siècle par un clerc breton nommé Maeloc, il renferme plusieurs collections canoniques irlandaises dont les prototypes ont été diffusés à

57. NORTIER, «Les bibliothèques médiévales... », p. 7. «Unde plurima aecclesiae nostrae proprio sudore conscripta reliquid volumina, id est volumen quatuor evangeliorum romana littera scriptum...» (Gesta Sanctorum Patrum..., éd. Fernand LOHIER et Jean LAPORTE, p. 89-90 ; éd. Pascal Pradié, p. 142-143). 
partir du monastère de Péronne, la version la plus proche du Bigotianus étant celle d'un manuscrit de Cambrai ${ }^{58}$. Ajoutons que la présence bretonne était particulièrement importante dans la région de Montreuil-sur-Mer et le Ternois au début du $\mathrm{X}^{\mathrm{e}}$ siècle, et cela en raison de l'arrivée de plusieurs groupes de réfugiés d'Armorique entre 913 et 920 .

58. Péronne: dép. Somme, chef-lieu de cant. PICARD, Jean-Michel, «L'Irlande et la Normandie avant les Normands (VII ${ }^{\mathrm{e}}$-XII ${ }^{\mathrm{e}}$ siècles) », Annales de Normandie, $47^{\mathrm{e}}$ année, $\mathrm{n}^{\circ}$ 1, mars 1997, p. 21-22. 